\title{
Clinical outcomes of lung adenocarcinoma patients harboring uncommon epidermal growth factor receptor (EGFR) mutations treated with EGFR-tyrosine kinase inhibitors (TKIs)
}

\author{
Jinfei $\mathrm{Si}^{1,2,3 \#}$, Xiaodong $\mathrm{Gu}^{1,2,3 \#}$, Wenxian Wang ${ }^{2,3 *}$, Shenpeng Ying ${ }^{4}$, Zhengbo Song ${ }^{2,3}$ \\ ${ }^{1}$ The Second Clinical Medical College of Zhejiang Chinese Medical University, Hangzhou, China; ${ }^{2}$ Department of Clinical Trial, Cancer Hospital \\ of the University of Chinese Academy of Sciences (Zhejiang Cancer Hospital), Hangzhou, China; ${ }^{3}$ Institute of Basic Medicine and Cancer (IBMC), \\ Chinese Academy of Sciences, Hangzhou, China; ${ }^{4}$ Department of Radiotherapy, Taizhou Central Hospital (Taizhou University Hospital), Taizhou, \\ China \\ Contributions: (I) Conception and design: Z Song; (II) Administrative support: Z Song; (III) Provision of study materials or patients: Z Song; (IV) \\ Collection and assembly of data: J Si; (V) Data analysis and interpretation: J Si, X Gu, W Wang; (VI) Manuscript writing: All authors; (VII) Final \\ approval of manuscript: All authors. \\ "These authors contributed equally to this work. \\ Correspondence to: Zhengbo Song. Department of Clinical Trial, Cancer Hospital of the University of Chinese Academy of Sciences (Zhejiang Cancer \\ Hospital), NO.1 Banshan East Road, Gongshu District, Hangzhou 310022, China. Email: songzb@zjcc.org.cn.
}

Background: This study aimed to explore the efficacy of different epidermal growth factor receptor tyrosine kinase inhibitors (EGFR-TKIs) in lung adenocarcinoma (AC) patients harboring uncommon EGFR mutations.

Methods: Between January 1st, 2013 and October 1st, 2019, 2,680 EGFR mutation-positive patients with confirmed stage IIIB/IV lung AC were enrolled from Zhejiang Cancer Hospital. Uncommon EGFR mutations were detected in 132 patients using next-generation-sequencing. Clinicopathological features between patients with uncommon EGFR mutations and common EGFR mutations were evaluated by the chi-square test. The clinical outcomes of patients with uncommon EGFR mutations were analyzed by the Kaplan-Meier method.

Results: Of 132 AC patients with uncommon EGFR mutations, 115 received EGFR-TKIs. Secondgeneration EGFR-TKIs were associated with longer progression-free survival (PFS) $(\mathrm{P}=0.116)$ and overall survival $(\mathrm{OS})(\mathrm{P}=0.005)$ than first or third-generation EGFR-TKIs. We also found that patients with compound mutations and double uncommon EGFR mutations had longer PFS $(\mathrm{P}=0.725)$ and $\mathrm{OS}(\mathrm{P}=0.741)$ than those with single uncommon $E G F R$ mutation, although the difference was not significant. In addition, third-generation EGFR-TKIs were more effective than the other two agents in patients with primary T790M mutation regarding PFS $(\mathrm{P}=0.150)$ and $\mathrm{OS}(\mathrm{P}=0.033)$, although the difference in PFS was not significant.

Conclusions: Patients with uncommon EGFR mutations treated with second-generation EGFR-TKIs showed better PFS and OS. EGFR-TKIs were more effective in patients with compound mutations or double uncommon mutations.

Keywords: Epidermal growth factor receptor (EGFR); uncommon mutation; compound mutation; double
uncommon mutation; lung adenocarcinoma (lung $\mathrm{AC}$ )

Submitted Sep 30, 2021. Accepted for publication Dec 16, 2021.

doi: 10.21037/apm-21-2828

View this article at: https://dx.doi.org/10.21037/apm-21-2828

\footnotetext{
^ ORCID: 0000-0001-6351-1690.
} 


\section{Introduction}

Lung cancer is the leading cause of death in China, and its incidence and mortality have increased in the last decade $(1,2)$. Non-small cell lung cancer (NSCLC) accounts for $85 \%$ of lung cancers (3), and lung adenocarcinoma (AC) is the most prevalent type of NSCLC. The epidermal growth factor receptor (EGFR) is involved in the proliferation, repair, and survival of tumor cells, and EGFR mutations are found in $40 \%$ of patients with NSCLC in Asian populations, which mainly occur in lung AC (4-6).

The development of targeted therapies has improved the survival of patients with lung AC (7). EGFR-sensitive mutations such as exon 19 deletions and $L 858 R$ point mutation are known as "classical" mutations (8), and patients with these mutations benefit from EGFR tyrosine kinase inhibitor (TKI) treatment (9-11). However, data on the efficacy of treatment in patients with uncommon $E G F R$ mutations in exon 18, exon 20, and exon 21, which account for $18 \%$ of EGFR mutations in NSCLC, is insufficient (12). The results of pooled analysis of the LUX-Lung 2, LUXLung 3, and LUX-Lung 6 clinical trials suggest that afatinib is effective in NSCLC patients carrying uncommon EGFR mutations (13). Cho et al. analyzed 37 NSCLC patients harboring uncommon $E G F R$ mutations $(G 719 X$, $L 861 Q, S 7681$ and others) and found that osimertinib had favorable activity in NSCLC patients harboring uncommon EGFR mutations (14). However, real-world data on the effects of EGFR-TKIs in patients with uncommon EGFR mutations are insufficient, and the efficacy of afatinib and osimertinib needs to be further validated. In addition, the clinical efficacy of these agents in patients with compound EGFR mutations and double uncommon mutations remains unclear. Kobayashi et al. analyzed 11 NSCLC patients with compound mutations or double uncommon mutations, and of eight patients treated with EGFR-TKIs, seven achieved a partial response (PR) with erlotinib (15). However, there are few studies analyzing compound mutations or double uncommon mutations. Hence, identifying effective treatment regimens for patients with compound mutations or double uncommon mutations is important.

The EGFR T790M point mutation in exon 20 is associated with acquired resistance to first or second generation EGFR-TKIs (16). Third-generation EGFRTKIs have been approved in many countries for patients with the EGFR T790M point mutation, in whom it prolongs progression-free survival (PFS) compared with platinum-based chemotherapy (17). However, primary
T790M mutations account for $0.5 \%$ of TKI-naïve NSCLC patients and survival data are lacking (18).

The development of next generation sequencing (NGS) has improved the detection of patients with uncommon $E G F R$ mutations and the treatment of this population needs to be addressed. Here, we performed a real-world study with a large number of patients to explore the efficacy of EGFRTKIs in AC patients harboring uncommon EGFR mutations. We present the following article in accordance with the STROBE reporting checklist (available at https://apm. amegroups.com/article/view/10.21037/apm-21-2828/rc).

\section{Methods}

\section{Study population}

This retrospective study was performed between January 1st, 2013 and October 1st, 2019. The study enrolled 2,680 EGFR mutation-positive patients with confirmed stage IIIB/ IV lung AC from Zhejiang Cancer Hospital. The study was performed in compliance with the ethical standards of Zhejiang Cancer Hospital (No. IRB-2021-440), and the need for written informed consent was waived because of the retrospective nature of the study. Of the 2,680 lung AC patients, some patients received first-line targeted therapies and other patients received second-line targeted therapies after experiencing disease progression with the standardized chemotherapy regimen. There were 2,548 patients with common EGFR mutations and 132 patients with uncommon EGFR mutations. All patients with uncommon EGFR mutations received EGFR-TKIs. The response to treatment was assessed according to Response Evaluation Criteria in Solid Tumors (RECIST v1.1). The study was conducted in accordance with the Declaration of Helsinki (as revised in 2013).

\section{Gene mutation detection}

Clinicopathological characteristics and gene abnormalities were analyzed by histopathological examination and routing mutation testing. NGS was performed to detect exon 19 deletions and L858R, L861Q, G719X, 20 insertions, S768I, and primary T790M mutations in accordance with the College of American Pathologists (CAP) guidelines $(19,20)$.

NGS detected 132 patients with uncommon mutations before EGFR-TKI treatment. Compound EGFR mutation was defined as the combination of a single uncommon 
Table 1 Clinicopathological features of uncommon EGFR mutation

\begin{tabular}{lcc}
\hline Items & Common EGFR mutation, $\mathrm{n}(\%)$ & Uncommon EGFR mutation, $\mathrm{n}(\%)$ \\
\hline Gender & $1,156(45.4)$ & $77(58.3)$ \\
Male & $1,392(54.6)$ & $55(41.7)$ \\
Female & & \\
Age, years & $1,387(54.4)$ & $66(50.0)$ \\
$\leq 60$ & $1,161(45.6)$ & $66(50.0)$ \\
$>60$ & & $68(52.7)$ \\
Smoking status & $1,685(68.0)$ & $61(47.3)$ \\
No & $793(32.0)$ & \\
Yes & & $110(83.3)$ \\
PS scores & $2,165(85.0)$ & $22(16.7)$ \\
$0-1$ point & $383(15.0)$ & 0.000 \\
$\geq 2$ points & & \\
\hline
\end{tabular}

EGFR, epidermal growth factor receptor; PS, performance status.

EGFR mutation and a sensitive EGFR mutation including exon 19 deletion and L858R point mutation. A double uncommon mutation was defined as the combination of two single uncommon mutations. The T790M mutation was considered a primary mutation as previous studies showed that an acquired T790M mutation was frequently present in patients who were resistant to first- or second-generation EGFR-TKIs.

\section{Statistical analysis}

Differences in clinicopathological features between patients with uncommon EGFR mutations and those with common EGFR mutations were evaluated by the chi-square test. The study had three endpoints: objective response rate (ORR), PFS, and overall survival (OS). ORR was defined as the sum of complete response and PR. PFS was defined as the time from the date of the start of EGFR-TKI treatment to the date of disease progression. OS was defined as the time from the initial diagnosis to the date of last followup or death from any cause. The latter two endpoints were analyzed using the Kaplan-Meier method. All analyses were performed using SPSS version 26 . Two-sided $\mathrm{P}$ values were set to $<0.05$. The last follow-up date was December 2020, and the median follow-up time was 27 months (range, 0.0 81.0 months).

\section{Results}

\section{Patient characteristics}

The 2,680 AC patients enrolled in the present study included 132 patients with uncommon EGFR mutations and 2,548 patients with common EGFR mutations. The clinicopathological features were analyzed and the clinical outcomes were evaluated until December 2020. Uncommon EGFR mutation patients accounted for $5 \%$ of the population and included 77 men and 55 women with a median age of 60 years (range, 29-84 years). Patients were divided according to smoking history into a never smoking group ( $\mathrm{n}=68)$ and former or current smoking group $(\mathrm{n}=61)$. The performance status was $0-1$ in 110 patients and $\geq 2$ in 22 patients. Uncommon mutations were more frequent in men $(\mathrm{P}=0.004)$ and in never smoking patients $(\mathrm{P}=0.000)$. Detailed patient characteristics are listed in Table 1.

\section{Survival analysis in patients with uncommon and common EGFR mutations}

Of the 2,680 stage IIIB/IV lung AC patients, 2,548 had common EGFR mutations and 132 had uncommon EGFR mutations. The uncommon EGFR mutations included G719X, S768I, insertion mutation, primary T790M mutation, and L861Q. The 132 lung AC patients with 
Table 2 Uncommon EGFR mutations were detected in lung adenocarcinoma patients by NGS

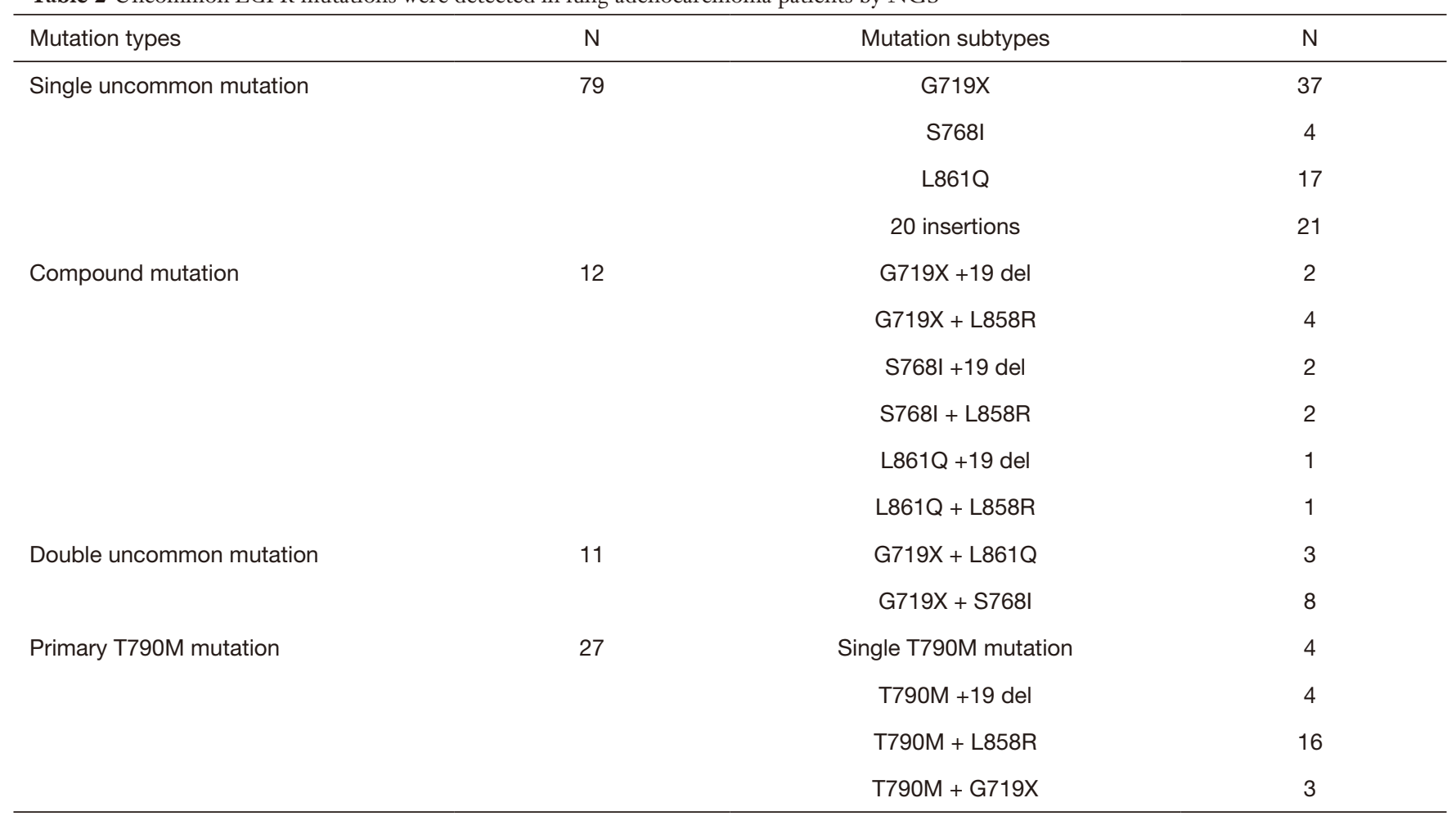

EGFR, epidermal growth factor receptor; NGS, next-generation sequencing.

uncommon $E G F R$ mutations were divided into four groups as follows: group 1: single uncommon $E G F R$ mutation (79/132); group 2: compound EGFR mutation (12/132); group 3: double uncommon EGFR mutation (11/132); and group 4: primary EGFR-T790M mutation (27/132). Three patients harboring exon 19 T751P mutation, exon 22 uncommon mutation, and exon 1 uncommon mutation were excluded from the analysis. Of 132 uncommon EGFR mutation patients, 115 received EGFR-TKIs (excluding three patients harboring other mutations), which consisted of first-generation EGFR-TKIs in 72 patients, secondgeneration EGFR-TKIs in 15 patients, and thirdgeneration EGFR-TKIs in four patients. The remaining 24 patients who received EGFR-TKIs were in the primary T790M mutation group, and this part of the population was analyzed separately. Uncommon EGFR mutations accounted for $5.0 \%$ of $E G F R$ mutations identified in the cohort, and the median PFS and OS were 8.2 and 27 months, respectively. Detailed information of patients with uncommon EGFR mutations is listed in Table 2.

Survival analysis of patients with uncommon and common mutations showed that patients harboring uncommon EGFR mutations (excluding primary T790M mutation) had shorter OS than those with common mutations (27 vs. 33 months, $\mathrm{P}=0.002$, Figure 1). Patients with uncommon mutations had a median OS of 27 months [95\% confidence interval (CI): 21.7-32.3 months] and an ORR of $13.2 \%(12 / 91)$.

\section{Treatment efficacy of EGFR-TKIs in patients harboring uncommon EGFR mutations}

Of the 132 uncommon EGFR mutation patients, the ORR of patients receiving first-generation EGFR-TKIs, secondgeneration EGFR-TKIs, and third-generation EGFR-TKIs was $9.7 \%$ (7/72), $26.7 \%$ (4/15), and 25\% (1/4), respectively. Patients (excluding group 4: primary EGFR-T790M mutation group) treated with second-generation EGFRTKIs $(n=15)$ had longer PFS than those treated with firstgeneration EGFR-TKIs ( $\mathrm{n}=72$ ) or third-generation EGFRTKIs $(\mathrm{n}=4)$, although the difference was not significant (9.0 vs. 6.5 vs. 1.9 months, $\mathrm{P}=0.116$, Figure 2). Patients treated with second-generation EGFR-TKIs $(\mathrm{n}=15)$ had longer OS than those treated with first- or third-generation EGFR- 


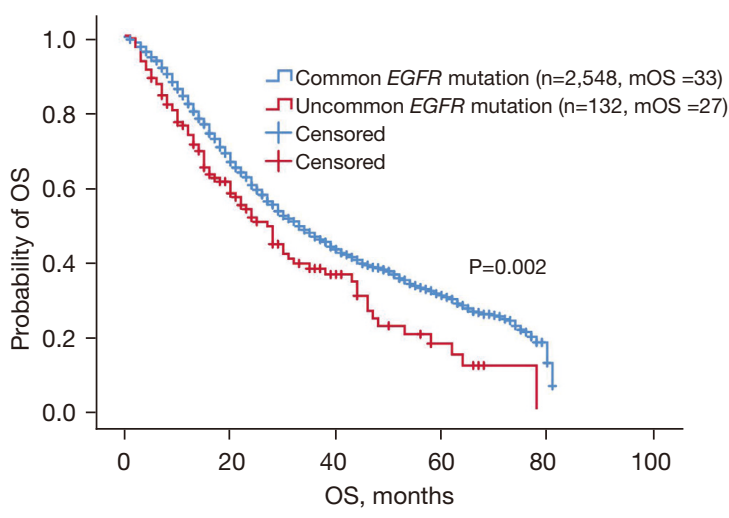

Figure 1 Uncommon EGFR mutation showed shorter OS than common EGFR mutation by Kaplan-Meier survival analysis. EGFR, epidermal growth factor receptor; OS, overall survival.

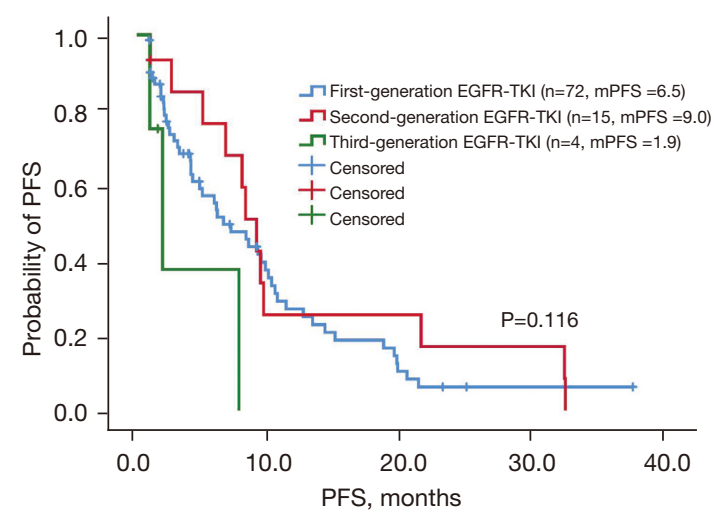

Figure 2 Second-generation EGFR-TKI showed longer PFS than first or third-generation EGFR-TKI by Kaplan-Meier survival analysis (excluding primary EGFR T790M mutation). EGFR-TKI, epidermal growth factor receptor-tyrosine kinase inhibitors; PFS, progression-free survival.

TKIs, and the difference was significant (NR vs. 20.4 vs. 13.8 months, $\mathrm{P}=0.005$, Figure 3). The median PFS and OS for patients treated with second-generation EGFR-TKIs was 9 months (95\% CI: 7.2-10.9 months) and not reached. The clinicopathological features of patients and the treatment efficacy of EGFR-TKIs are shown in Tables 3,4.

\section{Compound EGFR mutations and double uncommon EGFR mutations}

Of the 132 patients with uncommon EGFR mutations, 79 had a single uncommon EGFR mutation, 12 had compound $E G F R$ mutations (single uncommon $E G F R$

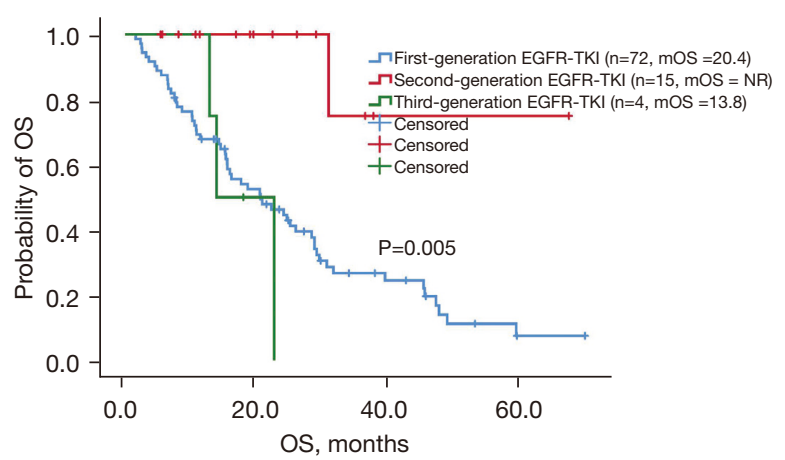

Figure 3 Second-generation EGFR-TKI showed longer OS than first or third-generation EGFR-TKI by Kaplan-Meier survival analysis (excluding primary EGFR T790M mutation). EGFRTKI, epidermal growth factor receptor-tyrosine kinase inhibitors; OS, overall survival.

mutation combined with sensitive EGFR mutation: exon 19 deletions and L858R point mutation), and 11 had double uncommon EGFR mutations. The ORR of patients with single uncommon mutation, compound mutation, and double uncommon mutation was $11.3 \%(8 / 71), 20 \%(2 / 10)$, and $20 \%(2 / 10)$, respectively. The median PFS of patients harboring single uncommon EGFR mutation, compound mutation, and double EGFR mutation was 6.7 months (95\% CI: 3.6-9.8 months), 9.0 months (95\% CI: 6.0 -12.0 months), and 9.4 months (95\% CI: 5.7-13.0 months), respectively. Patients harboring compound mutations and double $E G F R$ mutations had longer PFS than patients harboring single uncommon EGFR mutation, although the difference was not significant $(\mathrm{P}=0.725$, Figure 4). Patients with compound mutations and double uncommon EGFR mutations had longer OS than those with single uncommon EGFR mutations, with a median OS of 28 months (95\% CI: $14.3-41.7$ months) and 28 months (95\% CI: 14.9-41.1 months), respectively, and the difference was not significant $(\mathrm{P}=0.741$, Figure 5$)$. The median $\mathrm{OS}$ of patients with single uncommon EGFR mutation was 24 months (95\% CI: 15.2-32.8 months). The results suggested that EGFR-TKI treatment was more effective in patients with compound mutations and double uncommon $E G F R$ mutations than in those with single uncommon EGFR mutation.

\section{Primary T790M mutation}

Of 27 patients with primary EGFR T790M mutation (regardless of the type of mutation such as single 
Table 3 Clinicopathological features of different generation EGFR-TKI

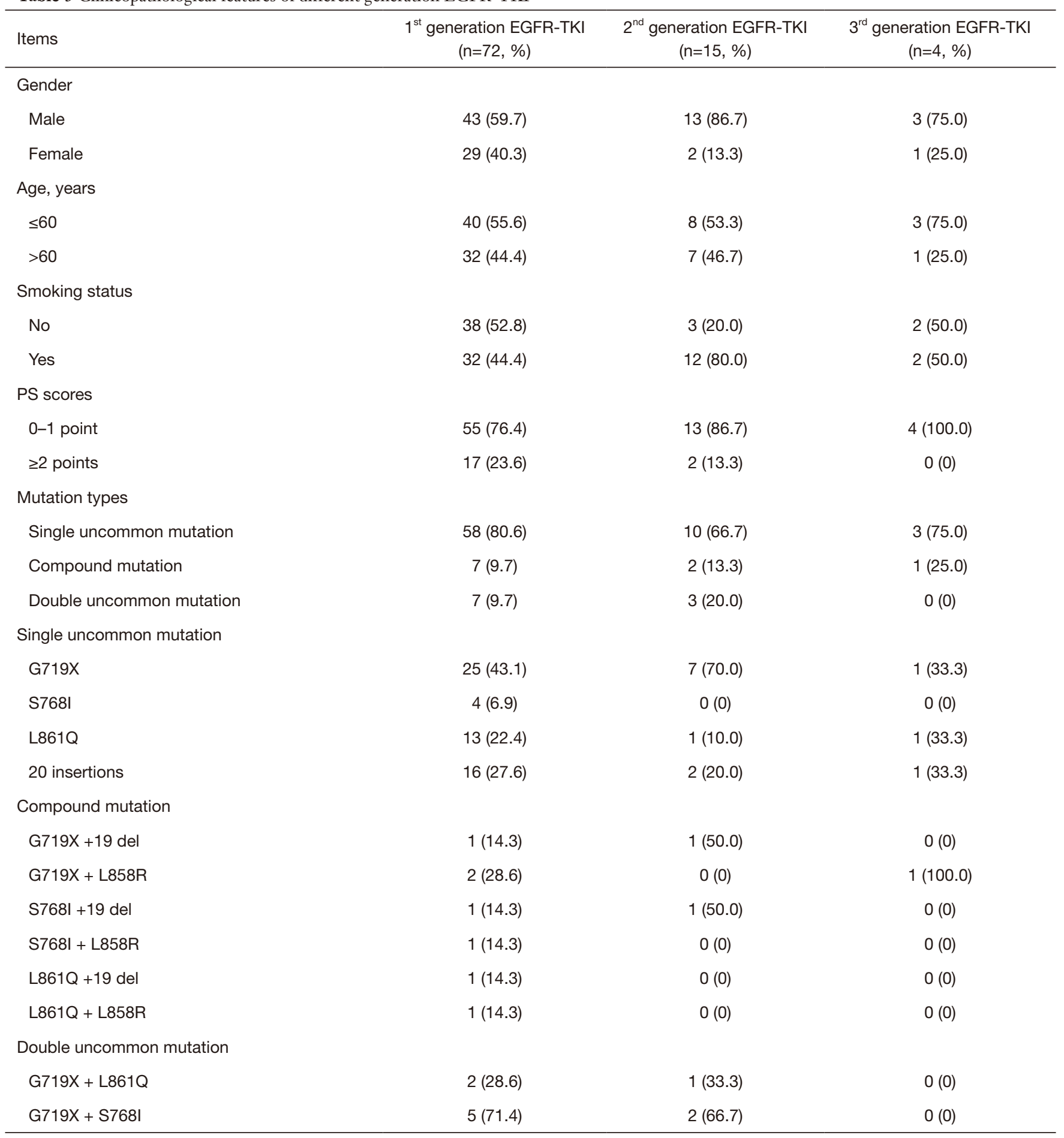

EGFR-TKI, epidermal growth factor receptor-tyrosine kinase inhibitors; PS, performance status. 
Table 4 The treatment efficacy of EGFR-TKI in lung adenocarcinoma patients harboring uncommon EGFR mutation (excluding primary T790M mutation)

\begin{tabular}{lcccc}
\hline EGFR-TKI & N & PFS (month, 95\% Cl) & P value & OS (month, 95\% Cl) \\
\hline $1^{\text {st }}$ generation EGFR-TKI (gefitinib or icotinib) & 72 & $6.5(3.6-9.4)$ & 0.116 & $20.4(11.9-29.0)$ \\
$2^{\text {nd }}$ generation EGFR-TKI (afatinib) & 15 & $9.0(7.2-10.9)$ & & NR \\
$3^{\text {rd }}$ generation EGFR-TKI (osimertinib) & 4 & $1.9(0.5-3.4)$ & $13.8(7.4-20.2)$ \\
\hline
\end{tabular}

The above data presented excluding primary T790M mutation group. EGFR-TKI, epidermal growth factor receptor-tyrosine kinase inhibitors; PFS, progression-free survival; OS, overall survival; NR, not reported.

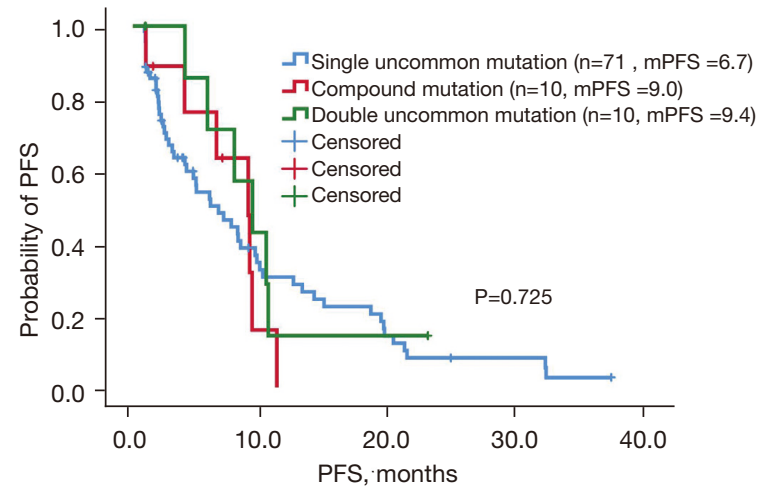

Figure 4 Compound mutation and double EGFR mutation had longer PFS than single uncommon EGFR mutation by KaplanMeier survival analysis (excluding primary EGFR T790M mutation). EGFR, epidermal growth factor receptor; PFS, progression-free survival.

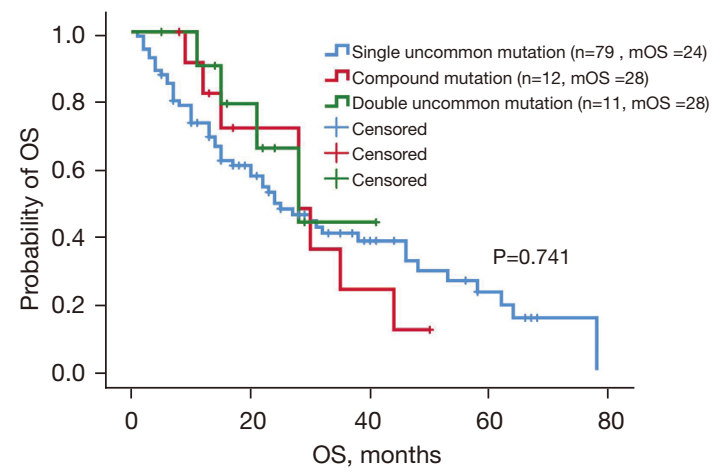

Figure 5 Compound mutation and double EGFR mutation had longer OS than single uncommon EGFR mutation by KaplanMeier survival analysis(excluding primary EGFR T790M mutation). EGFR, epidermal growth factor receptor; OS, overall survival. uncommon mutation, compound mutation, or double uncommon mutation), 12 were treated with first-generation EGFR-TKIs, 1 received second-generation EGFRTKIs, and 11 received third-generation EGFR-TKIs. The ORR of first-generation, second-generation, and thirdgeneration EGFR-TKIs was $0 \%(0 / 12), 0 \%(0 / 1)$ and $10 \%$ (1/10), respectively. There was no significant difference in PFS between these patients (8.2 vs. 9.5 vs. 12.2 months, $\mathrm{P}=0.150)$. However, patients treated with third-generation EGFR-TKIs had better PFS than those receiving the other two agents (median PFS $=12.2$ months, 95\% CI: 4.9-19.5 months). Patients treated with third-generation EGFR-TKIs had a significantly longer OS than those receiving first- or second-generation EGFR-TKIs (9.0 vs. 16.8 vs. 25.3 months, $\mathrm{P}=0.033$ ). The median $\mathrm{OS}$ of patients treated with third-generation EGFR-TKIs was 25.3 months (95\% CI: 7.6-43.0 months).

\section{Discussion}

Patients with uncommon EGFR mutations accounted for $5 \%$ of the population of this study and had a shorter OS than those with common mutations. Uncommon EGFR mutation patients treated with second-generation EGFRTKIs had longer PFS and OS than those treated with firstor third-generation EGFR-TKIs. The treatment efficacy of a second-generation EGFR-TKI (afatinib) was validated in a larger number of patients using real-world data. Patients with double uncommon EGFR mutations and compound mutations had longer PFS and OS than those with single uncommon EGFR mutations. The survival of patients harboring compound mutations or double uncommon mutations was examined in a larger sample size of patients.

The proportion of patients with uncommon EGFR 
mutations (5\% of EGFR mutations in our study) was smaller than that reported previously (21-24). In this study, patients with uncommon mutations who were treated with targeted therapies had shorter OS than patients with common mutations, with a median OS of 27 months. Kuiper et al. enrolled 54 NSCLC patients with nonclassic EGFR mutations and found that patients with nonclassic $E G F R$ mutations had a significantly shorter OS than those with classic EGFR mutations with a median OS of 20.2 months (25). Consistently, Chiu et al. showed that patients with uncommon EGFR mutations had a shorter OS than those with common EGFR mutations, with a median OS of 27.8 months (26). These results are consistent with those of the present study. Shen $e t$ al. showed that afatinib was effective in uncommon EGFR mutation patients, resulting in a median PFS of 11.0 months (27). Patients with uncommon EGFR mutations in this study could also benefit from EGFR-TKIs, although the treatment efficacy was lower than that in patients with common mutations. We therefore examined the differences in the efficacy of EGFR-TKIs in uncommon mutation patients.

Of 132 uncommon mutation patients, those treated with second-generation EGFR-TKIs had longer PFS than those receiving first- or second-generation EGFR-TKIs, with a median PFS of 9 months, although the difference was not significant. In addition, we found significant differences in OS between different generation EGFR-TKIs, as patients treated with second-generation EGFR-TKIs had a longer OS with a median OS not reached. Yang et al. reported that afatinib was effective in 75 NSCLC patients carrying uncommon EGFR mutations based on pooled analysis of the LUX-Lung 2, LUX-Lung 3, and LUX-Lung 6 clinical trials, with a median PFS of 10.7 months and a median OS of 19.4 months (13). A clinical trial including 315 NSCLC patients harboring uncommon EGFR mutations reported by Yang et al. demonstrated the efficacy of afatinib, with a median PFS of 10.8 months (28). Tanaka et al. enrolled 18 patients with uncommon EGFR mutations and showed that afatinib was associated with higher ORR and DCR than gefitinib or erlotinib (ORR: $75 \%$ vs. $40 \%$, DCR: $100 \%$ vs. $80 \%$ ); afatinib was also associated with longer PFS (17.1 vs. 5.5 months) (29). Lau et al. analyzed 16 uncommon mutation patients and found that the median OS of those treated with afatinib was 48.5 months and was longer than that of patients treated with gefitinib or erlotinib (30). The treatment efficacy of second-generation EGFR-TKIs was confirmed by Zhang et al. (31). We obtained similar results using a larger real-world sample size of uncommon
EGFR mutation patients to validate the treatment efficacy of second-generation EGFR-TKIs (afatinib). Cho et al. enrolled 37 patients in a clinical trial and found that osimertinib had favorable activity in NSCLC patients harboring uncommon EGFR mutations, with a median PFS of 8.2 months and the median OS was not reached (14). Ji et al. confirmed the efficacy of osimertinib in 51 uncommon mutations patients (32). However, in this study, the median PFS and OS of the four patients treated with osimertinib were 1.9 and 13 months, respectively, which was shorter than those of patients treated with afatinib. The difference between osimertinib and afatinib remained unclear because of the limitations of sample size.

Of the 132 patients with uncommon EGFR mutations, those with compound mutations and double uncommon EGFR mutations had longer PFS and OS than those with single uncommon EGFR mutation, although the difference was not significant. $\mathrm{Xu}$ et al. demonstrated that patients with compound mutations were sensitive to EGFR TKI therapy, with a median PFS of 9.79 months in 95 uncommon mutation patients (33). Chiu et al. analyzed 19 patients with double uncommon EGFR mutations and found significant differences in PFS between those with double uncommon EGFR mutations and those with single uncommon mutation (11.9 vs. 6.5 months; $\mathrm{P}=0.010$ ) (26). Passaro et al. analyzed 46 patients treated with EGFR-TKIs and found that Caucasian patients with compound mutations had better PFS and OS than patients without (PFS: 12.9 vs. 7.9 months; OS: 35.1 vs. 15.0 months) (34). This is consistent with the present results, although the difference between compound mutations and double uncommon mutations was not significant and needs to be further evaluated.

Among 27 patients with primary EGFR T790M mutation, those treated with third-generation agents had longer PFS and OS. Li et al. demonstrated that the primary EGFR T790M mutation frequently coexisted with L858R (18). This is consistent with the present study, in which the primary EGFR T790M mutation was more likely to coexist with L858R ( $\mathrm{n}=16)$ and accounted for $80 \%$ of EGFR T790M compound mutations. The present results suggesting that patients with primary T790M mutation could benefit from third-generation EGFR-TKIs (median PFS and OS of 12.2 and 25.3 months, respectively) are not consistent with previous studies. The efficacy of secondgeneration EGFR-TKIs towards the primary EGFR T790M mutation as an uncommon mutation remains unclear because of the limited sample size in this study. In addition, physicians give priority to third-generation 
EGFR-TKIs in daily practice regardless of the mutation type (primary or acquired T790M mutation).

Many factors affect OS outcomes, such as sample size and follow-up treatments. This study had several limitations, of which the main limitation was sample size. Other limitations are as follows: first, the primary data were obtained retrospectively, which may affect the results. Second, the sample size of patients with uncommon EGFR mutations was not adequate. Third, patients were recruited from a single institution, which may result in sampling bias and survival analysis bias.

In summary, patients with uncommon EGFR mutations had shorter OS than those with common EGFR mutations, and patients treated with second-generation EGFR-TKIs had better PFS and OS outcomes. Patients harboring compound mutations or double uncommon mutations showed better clinical outcomes than those with single uncommon mutation.

\section{Acknowledgments}

We would like to thank all the participants at the Second Clinical Medical College of Zhejiang Chinese Medical University, Zhejiang Cancer Hospital, Institute of Basic Medicine and Cancer (IBMC), and Chinese Academy of Sciences and Taizhou Central Hospital.

Funding: This work was supported by the Fund of the Science and Technology Department of Taizhou City (No. 1801ky36).

\section{Footnote}

Reporting Checklist: The authors have completed the STROBE reporting checklist. Available at https://apm. amegroups.com/article/view/10.21037/apm-21-2828/rc

Data Sharing Statement: Available at https://apm.amegroups. com/article/view/10.21037/apm-21-2828/dss

Peer Review File: Available at https://apm.amegroups.com/ article/view/10.21037/apm-21-2828/prf

Conflicts of Interest: All authors have completed the ICMJE uniform disclosure form (available at https://apm. amegroups.com/article/view/10.21037/apm-21-2828/coif). All authors report that they had support from the Fund of the Science and Technology Department of Taizhou City (No. 1801ky36).
Ethical Statement: The authors are accountable for all aspects of the work in ensuring that questions related to the accuracy or integrity of any part of the work are appropriately investigated and resolved. The study was conducted in accordance with the Declaration of Helsinki (as revised in 2013). The study was performed in compliance with the ethical standards of Zhejiang Cancer Hospital (No. IRB-2021-440), and the need for written informed consent was waived because of the retrospective nature of the study.

Open Access Statement: This is an Open Access article distributed in accordance with the Creative Commons Attribution-NonCommercial-NoDerivs 4.0 International License (CC BY-NC-ND 4.0), which permits the noncommercial replication and distribution of the article with the strict proviso that no changes or edits are made and the original work is properly cited (including links to both the formal publication through the relevant DOI and the license). See: https://creativecommons.org/licenses/by-nc-nd/4.0/.

\section{References}

1. Chen W, Zheng R, Baade PD, et al. Cancer statistics in China, 2015. CA Cancer J Clin 2016;66:115-32.

2. Pan R, Zhu M, Yu C, et al. Cancer incidence and mortality: a cohort study in China, 2008-2013. Int J Cancer 2017;141:1315-23.

3. D'Addario G, Früh M, Reck M, et al. Metastatic nonsmall-cell lung cancer: ESMO Clinical Practice Guidelines for diagnosis, treatment and follow-up. Ann Oncol 2010;21 Suppl 5:v116-9.

4. Shi Y, Au JS, Thongprasert S, et al. A prospective, molecular epidemiology study of EGFR mutations in Asian patients with advanced non-small-cell lung cancer of adenocarcinoma histology (PIONEER). J Thorac Oncol 2014;9:154-62.

5. Zhang YL, Yuan JQ, Wang KF, et al. The prevalence of EGFR mutation in patients with non-small cell lung cancer: a systematic review and meta-analysis. Oncotarget 2016;7:78985-93.

6. Gelatti ACZ, Drilon A, Santini FC. Optimizing the sequencing of tyrosine kinase inhibitors (TKIs) in epidermal growth factor receptor (EGFR) mutationpositive non-small cell lung cancer (NSCLC). Lung Cancer 2019;137:113-22.

7. Doroshow DB, Herbst RS. Treatment of advanced nonsmall cell lung cancer in 2018. JAMA Oncol 2018;4:569-70. 
8. Gazdar AF. Activating and resistance mutations of EGFR in non-small-cell lung cancer: role in clinical response to EGFR tyrosine kinase inhibitors. Oncogene 2009;28 Suppl 1:S24-31.

9. Mok TS, Cheng Y, Zhou X, et al. Improvement in overall survival in a randomized study that compared dacomitinib with gefitinib in patients with advanced non-small-cell lung cancer and EGFR-activating mutations. J Clin Oncol 2018;36:2244-50.

10. Park K, Tan EH, O’Byrne K, et al. Afatinib versus gefitinib as first-line treatment of patients with EGFR mutationpositive non-small-cell lung cancer (LUX-Lung 7): a phase 2B, open-label, randomised controlled trial. Lancet Oncol 2016;17:577-89.

11. Ramalingam SS, Vansteenkiste J, Planchard D, et al. Overall survival with osimertinib in untreated, EGFR-mutated advanced NSCLC. N Engl J Med 2020;382:41-50.

12. O'Kane GM, Bradbury PA, Feld R, et al. Uncommon EGFR mutations in advanced non-small cell lung cancer. Lung Cancer 2017;109:137-44.

13. Yang JC, Sequist LV, Geater SL, et al. Clinical activity of afatinib in patients with advanced non-small-cell lung cancer harbouring uncommon EGFR mutations: a combined post-hoc analysis of LUX-Lung 2, LUX-Lung 3, and LUX-Lung 6. Lancet Oncol 2015;16:830-8.

14. Cho JH, Lim SH, An HJ, et al. Osimertinib for patients with non-small-cell lung cancer harboring uncommon EGFR mutations: a multicenter, open-label, phase II trial (KCSG-LU15-09). J Clin Oncol 2020;38:488-95.

15. Kobayashi S, Canepa HM, Bailey AS, et al. Compound EGFR mutations and response to EGFR tyrosine kinase inhibitors. J Thorac Oncol 2013;8:45-51.

16. Yu HA, Arcila ME, Rekhtman N, et al. Analysis of tumor specimens at the time of acquired resistance to EGFR-TKI therapy in 155 patients with EGFR-mutant lung cancers. Clin Cancer Res 2013;19:2240-7.

17. Mok TS, Wu Y-L, Ahn M-J, et al. Osimertinib or platinum-pemetrexed in EGFR T790M-positive lung cancer. N Engl J Med 2017;376:629-40.

18. Li W, Qiu T, Guo L, et al. Primary and acquired EGFR T790M-mutant NSCLC patients identified by routine mutation testing show different characteristics but may both respond to osimertinib treatment. Cancer Lett 2018;423:9-15.

19. Pettersson E, Lundeberg J, Ahmadian A. Generations of sequencing technologies. Genomics 2009;93:105-11.

20. Yohe S, Thyagarajan B. Review of clinical next-generation sequencing. Arch Pathol Lab Med 2017;141:1544-57.
21. Kobayashi Y, Mitsudomi T. Not all epidermal growth factor receptor mutations in lung cancer are created equal: Perspectives for individualized treatment strategy. Cancer Sci 2016;107:1179-86.

22. Oxnard GR, Lo PC, Nishino M, et al. Natural history and molecular characteristics of lung cancers harboring EGFR exon 20 insertions. J Thorac Oncol 2013;8:179-84.

23. Yasuda H, Kobayashi S, Costa DB. EGFR exon 20 insertion mutations in non-small-cell lung cancer: preclinical data and clinical implications. Lancet Oncol 2012;13:e23-31.

24. Leventakos K, Kipp BR, Rumilla KM, et al. S768I mutation in EGFR in patients with lung cancer. J Thorac Oncol 2016;11:1798-801.

25. Kuiper JL, Hashemi SM, Thunnissen E, et al. Non-classic EGFR mutations in a cohort of Dutch EGFR-mutated NSCLC patients and outcomes following EGFR-TKI treatment. Br J Cancer 2016;115:1504-12.

26. Chiu CH, Yang CT, Shih JY, et al. Epidermal growth factor receptor tyrosine kinase inhibitor treatment response in advanced lung adenocarcinomas with G719X/ L861Q/S768I mutations. J Thorac Oncol 2015;10:793-9.

27. Shen YC, Tseng GC, Tu CY, et al. Comparing the effects of afatinib with gefitinib or Erlotinib in patients with advanced-stage lung adenocarcinoma harboring nonclassical epidermal growth factor receptor mutations. Lung Cancer 2017;110:56-62.

28. Yang JC, Schuler M, Popat S, et al. Afatinib for the treatment of NSCLC harboring uncommon EGFR mutations: a database of 693 cases. J Thorac Oncol 2020;15:803-15.

29. Tanaka I, Morise M, Kodama Y, et al. Potential for afatinib as an optimal treatment for advanced non-small cell lung carcinoma in patients with uncommon EGFR mutations. Lung Cancer 2019;127:169-71.

30. Lau SC, Chooback N, Ho C, et al. Outcome differences between first- and second-generation EGFR inhibitors in advanced EGFR mutated NSCLC in a large populationbased cohort. Clin Lung Cancer 2019;20:e576-83.

31. Zhang T, Wan B, Zhao Y, et al. Treatment of uncommon EGFR mutations in non-small cell lung cancer: new evidence and treatment. Transl Lung Cancer Res 2019;8:302-16.

32. Ji J, Aredo JV, Piper-Vallillo A, et al. Osimertinib in nonsmall cell lung cancer (NSCLC) with atypical EGFR activating mutations: a retrospective multicenter study. J Clin Oncol 2020;38:9570.

33. Xu J, Jin B, Chu T, et al. EGFR tyrosine kinase inhibitor 
(TKI) in patients with advanced non-small cell lung cancer (NSCLC) harboring uncommon EGFR mutations: A realworld study in China. Lung Cancer 2016;96:87-92.

34. Passaro A, Prelaj A, Bonanno L, et al. Activity of EGFR

Cite this article as: Si J, Gu X, Wang W, Ying S, Song Z. Clinical outcomes of lung adenocarcinoma patients harboring uncommon epidermal growth factor receptor (EGFR) mutations treated with EGFR-tyrosine kinase inhibitors (TKIs). Ann Palliat Med 2022;11(5):1624-1634. doi: 10.21037/apm-21-2828
TKIs in caucasian patients with NSCLC harboring potentially sensitive uncommon EGFR mutations. Clin Lung Cancer 2019;20:e186-e194. 